Revista

\title{
Multi-Ensayos
}

Vol. 6, núm. 11

ISSN: 2412-3285

https://multiensayos.unan.edu.ni

DOI: https://doi.org/10.5377/multiensayos.v6i11.9283

\section{El Cálculo Matemático aplicado a las Ciencias Económicas en el aula de clase}

\section{Mathematical Calculation Applied to Economic Sciences in the Classroom}

Donald Ariel Hernández Muñoz

Recibido: 20 de noviembre de 2019. Aceptado: 17 de enero de 2020

\section{RESUMEN}

El cálculo ha sido el instrumento elegido desde el principio, porque el concepto de «pequeñas variaciones» se encuentra en el centro de muchos problemas económicos. Este ensayo fue realizado con el propósito de analizar el cómo aprenden los estudiantes con perfil económico, algunos de los conceptos más importantes relacionados al Cálculo Matemático en el aula de clase, es decir reflexionar sobre algunos aspectos de la tarea de enseñar Matemáticas que resulta fundamental e importante, para promover mejores aprendizajes, de modo de seguir construyendo un marco interpretativo compartido sobre las características que debe asumir el trabajo matemático en el aula. Por ende, se tomado como muestra el segundo año de la carrera de Contaduría Pública y Finanzas de la UNAN - Managua, FAREM - Estelí.

Palabras claves: cálculo matemático; Ciencias Económicas; enseñar; aprendizaje.

\section{ABSTRACT}

The calculation has been the instrument chosen from the beginning, because the concept of "small variations" is at the center of many economic problems. This essay was carried out with the purpose of analyzing how students with an economic profile learn, some of the most important concepts related to Mathematical Calculation in the classroom, that is, to reflect on some aspects of the task of teaching Mathematics that is fundamental and important, to promote better learning, so as to continue building a shared interpretive framework on the characteristics that mathematical work in the classroom should assume. Therefore, it has been taken as a sample the public accounting and finance sophomores of the UNAN - Managua, FAREM - Estelí.

Keywords: mathematical calculation; Economic Sciences; teaching; learning processes.

1 Docente UNAN-Managua/FAREM-Estelí. Correo electrónico: hdonaldariel@gmail.com. (c) 2020 Revista Multi-Ensayos. 


\section{INTRODUCCIÓN}

"Enseñar exige respeto a los saberes de los educandos. Enseñar exige respeto a la autonomía del ser del educando. Enseñar exige seguridad, capacidad profesional y generosidad. Enseñar exige saber escuchar".

Paulo Freire.

Los estudiantes de Ciencias Económicas necesitan contar con fundamentos teóricos y conceptuales sobre el Cálculo Matemático que les permita mejorar sus procesos académicos. Por lo tanto analizar esas necesidades es el principal interés de este escrito.

¿Cómo aprenden los estudiantes con perfil económico, algunos de los conceptos más importantes relacionados al Cálculo Matemático en el aula de clase?

La interrogante anterior implica, que el presente ensayo tiene como fin reflexionar sobre algunos aspectos de la tarea de enseñar Matemáticas que resulta fundamental e importante, para promover mejores aprendizajes, de modo de seguir construyendo un marco interpretativo compartido sobre las características que debe asumir el trabajo matemático en el aula.

Además, se pretende describir las experiencias obtenidas en el aula de clase durante el curso de Matemática II, con estudiantes de la carrera de Contaduría Pública Y Finanzas de la Facultad Regional Multidisciplinaria, FAREM -Estelí.

La Matemática es lógica, precisa, rigurosa, abstracta, formal y bella. Representa un saber escalonado, donde cada etapa es necesaria para afrontar la siguiente, esto significa que debe aprobarse Matemática I para poder matricular Matemática II. Esta ciencia fortalece el pensamiento crítico para entender mejor el entorno, desarrolla la lógica de pensamiento para la toma de decisiones. (Martínez V, González Tellería, \& Rodríguez, 2013, pág. 4).

Tomando en consideración lo planteado anteriormente, en este documento se presentan algunos elementos que implican la reflexión para abordar la construcción de conceptos matemáticos en el aula de clase, y a manera de ejemplo, aplicaciones del Cálculo Matemático en las Ciencias Económicas. Además, se describen las fortalezas y dificultades presentadas por los estudiantes durante el desarrollo del proceso de enseñanza - aprendizaje.

\section{DESARROLLO}

La asignatura Matemática II contribuye al desarrollo de las capacidades esenciales como son: aplicar conceptos, definiciones, axiomas, propiedades y teoremas de derivadas parciales, integrales, ecuaciones diferenciales y matrices en la solución de ejercicios y problemas de la vida diaria; Adicionalmente favorece al logro de las capacidades de argumentación, análisis y crítica, precisión, rigor, formalización, creatividad, intuición, lógica de pensamiento y toma de decisiones adecuadas. (Martínez V, González Tellería, \& Rodríguez, 2013, pág. 5). 
Como docente de Matemáticas a lo largo de estos años de experiencia, también he sido testigo de un fenómeno triste que pasa con los estudiantes y es que estos tienden a frustrarse ante el cálculo no porque encuentren que el tema es imposible, sino porque tienen habilidades superficiales en la Matemática general y en específico en la unidad de álgebra y trigonometría.

Lo anterior implica, que en los cursos de Cálculo construyen de inmediato sobre su conocimiento y habilidades previos, donde hay mucho terreno nuevo por cubrir. En consecuencia, hay poco tiempo para repasar las bases en el planteamiento formal dentro de las aulas de clase.

Así, quienes enseñamos cálculo debemos asumir que usted puede factorizar, simplificar y resolver ecuaciones, desigualdades, manejar valores absolutos, usar una calculadora, aplicar las leyes de los exponentes, encontrar ecuaciones de rectas, trazar gráficas elementales y aplicar importantes identidades logarítmicas y trigonométricas, la habilidad de hacer álgebra y trigonometría, trabajar con exponentes y logaritmos, así como trazar a mano, con rapidez y precisión, gráficas básicas que son claves para tener éxito en un curso de cálculo. (G. Zill \& S. Wright., 2011)

Por ende, teniendo como referencia lo antes planteado, en cada inicio de curso que se comparte con estudiantes ya sean de carreras de Ciencias de la educación, de Tecnología o de Ciencias Económicas, se inicia no solo con guías diagnósticas de matemática básica, sino que se comienza con la concientización o reflexión de:

"No asumir que pueden lograrlo con un esfuerzo mínimo porque identifican algunos de los temas en cálculo diferencial e integral. Un sentimiento de familiaridad con el tema en estudio, combinado con una actitud de complacencia a menudo es la razón de resultados no muy satisfactorios al final."

Tal como dice (G. Zill \& S. Wright., 2011), aprender matemáticas no es como aprender a andar en bicicleta: en que una vez que se aprende, la habilidad permanece para siempre. Las matemáticas son más como aprender otro idioma o tocar un instrumento musical: requiere tiempo, esfuerzo y mucha práctica para desarrollar y mantener la habilidad. Aun los músicos experimentados continúan practicando escalas fundamentales.

Según, (Villa Ochoa, 2015, pág. 135), uno de los argumentos por los que se recomienda la implementación de la modelación matemática como recurso en las aulas escolares es el hecho de que, a partir de ella, las matemáticas pueden observarse como una herramienta que permite describir y analizar algunos fenómenos o problemas del entorno en el cual se desenvuelven los individuos cotidianamente.

A continuación se presentan algunas temáticas que se abordan en los cursos de Cálculo, aplicadas a las Ciencias Económicas en el aula de clase (ejemplo, con la carrera de Contaduría):

\section{Límites y continuidad}

1. Aplicaciones de límites - Interés compuesto en forma continua.

2. Límites y continuidad - Aplicación práctica - Déficit de presupuesto. 


\section{Aplicaciones de la diferenciación}

3. Diferenciales y Elasticidad de demanda.

\section{Integración}

- El Teorema fundamental del Cálculo Integral, área entre curvas...

4. Excedentes de consumidores y fabricantes. (integración).

5. Aplicación práctica - Precio de un artículo vendido.

6. Aplicación de integrales en la distribución de ingresos, Curva de Lorenz e Índice de Gini que mide el grado de la distribución de la renta (o del consumo) entre los individuos de un país con respecto a una distribución con perfecta igualdad.

\section{Cálculo en varias variables}

7. Análisis de datos para modelar el enfriamiento.

8. Las matrices de insumo-producción o insumo-producto (Análisis).

9. Amortización de créditos y la regla de los 78 .

10. Aplicaciones de las ecuaciones diferenciales en las ciencias económicas.

Según (Cámara Sánchez, pág. 105), el instrumento matemático más útil para el economista es el cálculo diferencial. Trata esencialmente las tasas de variación y es el instrumento natural que emplea el economista en la construcción y discusión de teorías económicas. Los economistas están más interesados en las cantidades marginales que en las cantidades totales.

El cálculo integral también encuentra fácil aplicación a los problemas económicos, especialmente en los campos de la organización industrial y de la hacienda pública. El álgebra matricial se ha mostrado especialmente útil en el tratamiento de las grandes cantidades de ecuaciones y variables que resumen las de la economía del mundo real.

El cálculo ha sido el instrumento elegido desde el principio, porque el concepto de «pequeñas variaciones» se encuentra en el centro de muchos problemas económicos.

Por ejemplo, un medio común de calcular los beneficios consiste en el cálculo del excedente de los consumidores, es decir, los precios máximos que los consumidores estarían dispuestos a pagar por el bien o servicio. La medida matemática de este beneficio agregado es la integral (la suma) de los beneficios de todos los individuos bajo la curva de demanda del bien en cuestión. (Cámara Sánchez, pág. 106).

Por ende, estas asignaturas se desarrollan aplicando una metodología participativa, basada en el aprender - haciendo. Para ello, se aplican técnicas, tales como: lluvias de ideas, explicación participativa, aprendizaje basado en preguntas dirigidas, aprendizaje por tareas, participación en la pizarra, pruebas escritas, investigaciones, aprendizaje colaborativo (trabajo en equipo), exposiciones, debates, aprendizaje basado en la resolución de ejercicios y problemas. 
Lo antes mencionado, permite un proceso pedagógico activo que integra la teoría con la práctica. Dado que es importante el protagonismo de los estudiantes, el diálogo de saberes, intercambio de experiencias y la producción colectiva de aprendizajes en la interacción entre el estudiantado y facilitador, es una metodología muy importante ante el aprendizaje de conceptos matemáticos y que luego a la aplicación en los estudios de caso relacionados a la vida diaria.

Dando salida a la interrogante planteada en la introducción de, ¿cómo aprenden los estudiantes con perfil económico, algunos de los conceptos más importantes relacionados al Cálculo Matemático en el aula de clase?, además de lo descrito en los párrafos anteriores, se plantea a continuación el cómo "hacer matemáticas".

En los programas de Cálculo de la UNAN -Managua, se contempla el modo de hacer y pensar de la matemática como característica del trabajo que se debe promover en las aulas. Esto conlleva a pensar que "se aprende matemática haciendo matemática", lo que involucra pensar la clase como un espacio de producción que guarde semejanza con el quehacer de la disciplina.

Al reflexionar sobre cómo hacer matemáticas, claro está que desarrollar un trabajo de esta naturaleza está profundamente ligado con las decisiones del docente al planear la clase, así como en sus intervenciones durante el proceso de enseñanza. Esto conlleva a pensar desde este punto de vista, cómo tratar la clase para favorecer este trabajo matemático.

Para sintetizar el cómo enseñar el Cálculo Matemático en las aulas de clase, en manera de ejemplo se presenta el relato de una sesión de clase, centrándose en el análisis de algunas condiciones didácticas ligadas a la gestión o planeación por parte del docente.

La situación corresponde a un segundo año de la carrera de Contaduría Pública y Finanzas, en el análisis de las aplicaciones de la integral definida. La clase se inicia con el análisis de definiciones y teoremas sobre cálculo de áreas bajo curvas y áreas entre funciones, para luego entrar a las aplicaciones de estas temáticas.

El docente propone a reflexionar en la resolución de problemas, en esta instancia los estudiantes, por medio del aprendizaje por tareas, ya han indagado sobre, costo marginal, total, demanda, distribución de ingresos (Curva de Lorenz, índice de Gini e igualdad de ingresos), entre otros.

En esta etapa, se recalca que en sesiones anteriores han surgido diferentes estrategias para resolver problemas de aplicación, y que les propone una nueva serie de situaciones, para seguir pensando, y más que eso vallan valorando la gran aplicación de las matemáticas en lo económico.

En forma de ejemplo se copia en la pizarra problemas de aplicación y les propone que cada uno resuelva en su cuaderno de manera individual, como les parezca, y que anoten, cómo lo realizan. 
1. "La definición de costo marginal de un fabricante es: la función de costo total es: $d c / d q=0.6 q+2$. Si la producción actual es $q=80$ unidades por semana, ¿cuánto más costará incrementar la producción a 100 unidades por semana?

2. La distribución de cierto País está descrita por la Curva de Lorenz $f(x)=20 / 21 x^{2}+1 / 21 x$.

a) ¿Qué proporción de los ingresos totales del País recibe el $50 \%$ de la población más pobre?

a) Determine el Coeficiente de desigualdad correspondiente a esta curva". (Haeussler, Richard S, \& Richard J, 2008)

Resulta interesante analizar que si bien los problemas solo consisten en resolver un cálculo, se trata de una situación que requiere análisis para estos estudiantes. Ellos deberán elegir una estrategia entre varias posibles y dar cuenta de por qué realizaron el cálculo de esa manera. Esta misma actividad puede no resultar tan complicada para algunos, para los cuales se trata de un ejercicio que no les opone resistencia. Mientras los estudiantes resuelven, el facilitador recorre el aula mirando el avance de la actividad y del cómo la hacen. Propone un clima de búsqueda, para lo cual no da pistas sobre cómo se resuelven los problemas.

Cuando los educandos tienden a preguntar si está bien lo que hicieron o lo que están realizando hasta un momento determinado, el docente no indica si es correcto o no, sino que les propone esperar un rato. Si el grupo de estudiantes no encuentra la manera de resolver, les propone que revisen en el cuaderno cómo resolvieron cálculos similares. Durante esta fase de trabajo, observa que los estudiantes han desplegado diversas estrategias de resolución.

Este ambiente, permite que el rol de los estudiantes, según la (UNAN - Managua, 2011, pág. 46), en su modelo educativo que, el proceso educativo que se desarrolla en la universidad concibe la formación de la personalidad del discente respetando su autonomía, su individualidad y la capacidad de decisión. Esto a su vez, le confiere un rol más activo y reflexivo con el propósito de poder interiorizar los conocimientos y experiencias educativas de forma significativa.

Lo antes planteado, se traduce en un aprendizaje pertinente que responde a las demandas personales y sociales. El estudiante asume un compromiso genuino con su formación, es decir, es participativo, dinámico, creativo y crítico del proceso de enseñanza-aprendizaje en todas sus dimensiones.

Siguiendo con la descripción, antes de poner en discusión los distintos procedimientos, el docente decide proponer una nueva actividad, con el propósito de generar mejores condiciones para la puesta en común que reunidos en trio verifique los resultados obtenidos, la posibilidad de debatir con los compañeros que usaron la misma o distinta metodología de solución les da la posibilidad de llegar al momento de trabajo colectivo al reflexionar sobre lo realizado.

Sin embargo, no es suficiente para asegurar que la estrategia utilizada es la correcta. Hay que tratar de aplicar otros medios que certifiquen que el procedimiento seguido es válido, lo que será tratado en la puesta en común. 
Una vez finalizada la fase de resolución individual y la corrección en pequeños equipos, el facilitador propone un intercambio colectivo de manera general, esto implica por un lado en compartir los diferentes procedimientos y que los estudiantes relaten en qué se han equivocado y por qué; por otro, que planteen el por qué lo resolvieron así y en qué basaron sus decisiones.

"El éxito total nunca es alcanzado por un solo individuo, siempre hay un grupo de personas detrás de él." (T. Harv Eker)

Para concluir con una sesión de clase de matemática, el facilitador realiza de manera conjunta una síntesis de los conceptos abordados. Se trata de un momento de sistematizar, de modo que lo surgido en torno a la resolución de problemas adquiera un mayor nivel de generalidad y permita resolver una familia de problemas de aplicación del mismo tipo. Las estrategias y conocimientos sistematizados podrán reutilizarse para resolver otros problemas.

En el momento de enseñar un determinado contenido se pueden llevar a cabo distintas propuestas de enseñanza. Las decisiones que toma el docente en torno a qué problemas seleccionar, qué tipo de resoluciones aceptar y qué debates promover, entre otras características que asume la gestión de la clase, configuran distintas enseñanzas, que impactan directamente en lo que los estudiantes van a aprender.

Para finalizar, es importe recalcar que, mediante el trabajo colaborativo (trabajo en equipo), se observó que no necesariamente los estudiantes que dominan más, han logrado buenos resultados, sino también aquellos que lograron organizarse, aprovechando sus fortalezas y oportunidades.

\section{CONCLUSIÓN}

En este apartado se presenta una síntesis de las conclusiones a las que se llegó después de culminar el curso de Matemática II, con estudiantes del segundo año de Contaduría Pública y Finanzas de la UNAN - Managua, FAREM - Estelí, acorde al cumplimiento de los fines planteados.

A continuación se precisan las principales conclusiones de este escrito:

El instrumento matemático más útil para el economista es el cálculo diferencial. El cálculo integral también encuentra fácil aplicación a los problemas económicos, especialmente en los campos de la organización industrial y de la hacienda pública.

El trabajo individual, colaborativo y cooperativo aportan al estudiante un tipo de interacción diferente con el conocimiento, por lo tanto todas son necesarias en la clase.

Una matemática aplicada al perfil de la carrera, genera en el estudiantado el placer por aprender y confianza en las propias posibilidades, impactando positivamente en el desempeño de cada uno. 
Durante el proceso de enseñanza - aprendizaje de la asignatura, se construyeron los aprendizajes partiendo de los saberes previos, a través de los métodos y técnicas.

La buena planificación de la Matemática aplicada a una carrera de perfil económico, conlleva al estudiantado a ser sujetos activos, participativos, investigativos y constructores de su propio conocimiento.

En el proceso de enseñar el Cálculo se promueve la reflexión mediante técnicas, como la resolución de problemas, los debates y el análisis de casos.

Cabe destacar que en la asignatura, hubo un 100\% de aprobación, lo que prueba el nivel de aprendizaje de los estudiantes. Por todo lo expuesto en este ensayo, se puede reiterar que la experiencia, fue positiva tanto para estudiantes y docente.

Por lo anterior, el estudiante, sólo puede aprender matemáticas (es decir, hacer "que se le pegue") mediante el trabajo arduo de hacer matemáticas. Tal como plantea (G. Zill \& S. Wright., 2011), en su libro de Cálculo, "No puede leer un texto de este tipo como si fuese una novela; debe abrirse camino a lo largo de él con lápiz y papel en mano.

\section{BIBLIOGRAFÍA}

Cámara Sánchez, Á. (2000). Aportaciones de la matemática a la metodología económica. Psicothema, XII, 103-107. Obtenido de http://www.redalyc.org/articulo.oa?id=72797027

G. Zill, D., \& S. Wright., W. (2011). tempranas, CÁLCULO Trascendentes (Cuarta edición ed.). México, D. F.: The McGraw-Hill Companies, Inc.

Haeussler, E. F., Richard S, P., \& Richard J, W. (2008). Matemáticas para Administración y Economía (Decimosegunda ed.). México: PEARSON EDUCACIÓN.

Martínez V, S., González Tellería, M., \& Rodríguez, F. (2013). Programa de Asignatura Matemática II. Managua, Nicaragua: UNAN-Managua.

UNAN - Managua. (2011). Modelo Educativo, Normativa y Metodología para la Planificación Curricular 2011. Managua: UNAN - Managua.

Villa Ochoa, J. A. (2015). Modelación matemática a partir de problemas de enunciados verbales: un estudio de caso con profesores de matemáticas. Magis, VIII, 133-148. Obtenido de http://www.redalyc. org/articulo.oa?id=281042327008 